International Journal of Modern Physics C,

(C) World Scientific Publishing Company

\title{
PATTERNS AND LONG RANGE CORRELATIONS IN IDEALIZED GRANULAR FLOWS
}

\author{
J.A.G. ORZA and R. BRITO \\ Facultad de Ciencias Físicas, Universidad Complutense \\ 28040 Madrid, Spain \\ and \\ T.P.C. VAN NOIJE and M.H. ERNST \\ Instituut voor Theoretische Fysica, Universiteit Utrecht, Postbus 80006 \\ 3508 TA Utrecht, The Netherlands \\ Received February 1, 2008 \\ Revised (revised date)
}

\begin{abstract}
An initially homogeneous freely evolving fluid of inelastic hard spheres develops inhomogeneities in the flow field $\mathbf{u}(\mathbf{r}, t)$ (vortices) and in the density field $n(\mathbf{r}, t)$ (clusters), driven by unstable fluctuations, $\delta a=\{\delta n, \delta \mathbf{u}\}$. Their spatial correlations, $\left\langle\delta a(\mathbf{r}, t) \delta a\left(\mathbf{r}^{\prime}, t\right)\right\rangle$, as measured in molecular dynamics simulations, exhibit long range correlations; the mean vortex diameter grows as $\xi(t) \propto \sqrt{\ln t}$; there occur transitions to macroscopic shearing states, etc.

The Cahn-Hilliard theory of spinodal decomposition offers a qualitative understanding and quantitative estimates of the observed phenomena. When intrinsic length scales are of the order of the system size, effects of physical boundaries and periodic boundaries (finite size effects in simulations) are important.
\end{abstract}

\section{Introduction}

Standard fluids, when out of equilibrium, will rapidly decay to local equilibrium within a few mean free times. The subsequent decay of spatial inhomogeneities towards global equilibrium is controlled by the slow hydrodynamic time evolution. A prototypical model for this is a system of $N$ smooth elastic hard spheres. Compare this system to a system of smooth inelastic hard spheres, defined such that in the center of mass frame a fraction of order $\epsilon=1-\alpha$ (where $\alpha$ is called the restitution coefficient; $0<\alpha<1$ ) of the kinetic energy is lost in each collision (for precise definitions we refer to Ref. 1). Linear momentum is conserved during collisions. Consequently the local momentum density, $\mathbf{g}(\mathbf{r}, t)=n(\mathbf{r}, t) \mathbf{u}(\mathbf{r}, t)$, or the flow velocity $\mathbf{u}(\mathbf{r}, t)$ is a slowly varying quantity, and the system can still be considered a fluid. The lack of energy conservation makes this fluid, whether driven by gravity or shear stresses, or freely evolving, behave very differently from a standard fluid $\mathrm{B}$

The system of inelastic hard spheres represents an idealized model for rapid granular flows, where the dynamics of individual (macroscopic) particles is described by binary collisions, separated by free propagation over a typical mean free path. 
To study this problem by computer simulations, we consider a 2-dimensional system of $N$ inelastic hard disks of diameter $\sigma$ and unit mass, contained in a volume $V=L^{2}$ with periodic boundary conditions, and use an event-driven molecular dynamics code. 3 At the initial time the system starts off in a spatially homogeneous equilibrium state of elastic hard disks $(\epsilon=0)$. From there on the kinetic energy is dissipated by collisions, which leads to cooling.

We monitor the time evolution of the system, and measure the velocity distribution of a particle, the time dependence of the kinetic energy, and the total number of collisions. These are the standard quantities that can be obtained from the Enskog-Boltzmann equation.

The novel feature of the present study is an analysis of the spatial correlations in mass and energy densities, and in flow velocities at different points in the system, which exhibit long range spatial correlations. To understand such dynamic correlations one has to go beyond the standard Enskog-Boltzmann equation, which is based on the molecular chaos assumption, and use the Ring kinetic theory, which accounts for sequences of correlated binary collisions. Ring kinetic theory and closely related mode coupling theories have been succesful in explaining the phenomena of long time tails, and of long range spatial correlations in nonequilibrium stationary states, violation also occurs in systems of inelastic hard spheres.

In fact, we have applied the Ring theory to such systems, and succesfully explained the behavior of the nonequilibrium pair distribution at late times and large distances. Preliminary results of this investigation have been reported at several conferences and workshops.

The plan of this paper is as follows: section 2 describes the homogeneous cooling state. Spatial fluctuations (section 3) drive the system away from this state through slow hydrodynamic modes (section 4). Some modes are unstable and lead to phase separation and clustering (section 5). Vorticity diffusion controls the long time behavior (section 6) and leads to scaling laws. Section 7 deals with the effects of boundaries on the structure of asymptotic states.

\section{Homogeneous Cooling State}

What are the important observations that can be deduced from molecular dynamics simulations, performed on a system of $N$ inelastic hard disks, that are prepared in a spatially homogeneous equilibrium state of an elastic hard disk system?

The system stays for many collision times in the so-called homogeneous cooling state, described by a single particle distribution function which is essentially a Maxwellian with a time dependent total kinetic energy $N E(t)$. 2 .. . As long as the system is in this homogeneous cooling state, the energy per particle equals the temperature, $T(t)=E(t)$. This temperature decays as $t^{-2}$ for long times (see Fig. 1), in quantitative agreement with the predictions from kinetic theory, and has the form

$$
T(t) \equiv \frac{1}{2} v_{0}^{2}(t)=T(0) /\left[1+t / t_{e}\right]^{2}
$$




$$
=T(0) \exp \left[-2 \gamma_{0} \tau\right]
$$

Here $\gamma_{0}=\frac{1}{4}\left(1-\alpha^{2}\right)=\frac{1}{2} \epsilon\left(1-\frac{1}{2} \epsilon\right)$ measures the degree of inelasticity, and $v_{0}(t)$ is the thermal velocity. The characteristic time of homogeneous cooling, $t_{e}=t_{0} / \gamma_{0}$, is proportional to the mean free time for elastic hard disks in the initial state, $t_{0}=1 / \omega_{0}(0)=1 /\left[\sqrt{2 \pi} n \chi \sigma v_{0}(0)\right]$, where $\chi$ is the radial distribution function for elastic hard disks at contact. With this definition, the mean free path at the initial time equals that of the elastic hard disks and is given by $l_{0}=v_{0}(0) t_{0}$. The average number of collisions $\tau$, suffered by a single particle in a time $t$, follows by integrating $d \tau=\omega_{0}(t) d t$, where $\omega_{0}(t)$ is the collision frequency of inelastic hard disks, which is proportional to $\sqrt{T(t)}$. The result is

$$
\gamma_{0} \tau=\ln \left[1+t / t_{e}\right]
$$

where $\tau$ is related to the total number of collisions $C$ as $C=\frac{1}{2} \tau N$.
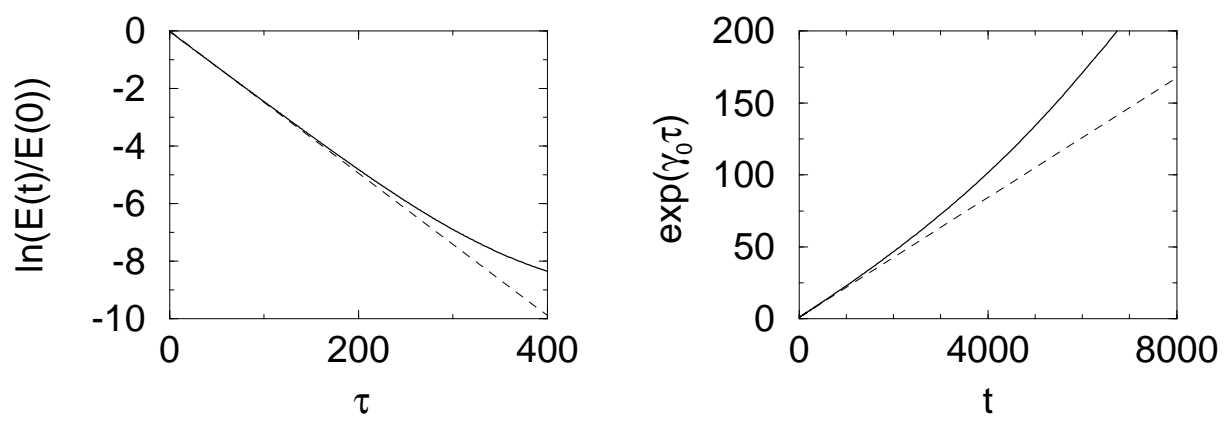

Fig. 1. Left: Plot of the logarithm of the total energy as a function of the number of collisions per particle $\tau$ in a simulation with $N=20000$ particles, system size of $L=253 \sigma$, and dissipation parameter $\epsilon=1 / 40$. The straight (dashed) line with slope $-2 \gamma_{0} \simeq-0.0247$ (Eq. (1)) corresponds to the behavior of the homogeneous cooling state. Deviations of $E(t)$ from the straight line around $\tau=200-250$ are correlated with the appearance of inhomogeneities in the density field (see behavior of $G_{n n}$ for $\tau=170-332$ in Fig. 2). Right: Number of collisions per particle $\tau$ versus time $t$ for the same system. The straight dashed line correponds to the homogeneous cooling prediction, Eq. (2).

In the subsequent time evolution of the inelastic hard disk system, the homogeneous cooling state plays a role very similar to local equilibrium in ordinary fluids, and provides a conceptual basis for a hydrodynamic description. However, this homogeneous cooling state does not at all behave as an equilibrium state of $N$ elastic hard disks with a time dependent temperature. In the latter state spatial correlations are caused by hard core excluded volume effects and extend only over a few disk diameters $\sigma$. In the present system of inelastic hard spheres, however, we have observed: (i) long range spatial correlations extending over more than a decade of disk diameters; (ii) slowly growing spatial inhomogeneitiest. 10 in the local flow field $\mathbf{u}(\mathbf{r}, t)$ and density field $n(\mathbf{r}, t)$, which can be described by coupling of hydrodynamic modes, at least for times that are not too long; (iii) a collision number $\tau$ that 
increases more rapidly than $\left(1 / \gamma_{0}\right) \ln \left(1+t / t_{e}\right)$, and a cooling law that decays more slowly 10 than the prediction of Eq. (1), which is illustrated in Fig. 1.

\section{Spatial Correlations}

To obtain a better understanding of the dynamics of a freely evolving system of these inelastic hard disks, we follow the local mass density $n(\mathbf{r}, t)$, the local momentum density $\mathbf{g}(\mathbf{r}, t)=n(\mathbf{r}, t) \mathbf{u}(\mathbf{r}, t)$ and the local energy per particle $e(\mathbf{r}, t)$ in a single realization of a system of $N=20000$ particles by means of computer simulations, where the inelasticity parameter is $\epsilon=1 / 40$ and the volume fraction $\phi=\frac{1}{4} \pi n \sigma^{2} \simeq$ 0.245 , corresponding to a system size $L \simeq 253 \sigma$.

Visual observation of snapshots of $n(\mathbf{r}, t)$ - and $\mathbf{g}(\mathbf{r}, t)$-fields show that at early times $(\tau=82)$, the momentum density does not exhibit much visible structure, and the mass density is totally homogeneous. At $\tau=120-170$ the mass density is still homogeneous, but the flow field starts to show vortices after coarse graining over cells of $5 \sigma \times 5 \sigma$. At the latest time $(\tau=332)$ the flow field shows a pronounced structure of vortices, also in the fine grained $\mathbf{u}(\mathbf{r}, t)$-field, with a typical diameter $\xi$ of the order of $\frac{1}{4}$ of the system size $L$, and the mass density starts to show barely visible inhomogeneities in the fine grained $n(\mathbf{r}, t)$-field, but clearly visible in the coarse grained $n(\mathbf{r}, t)$-fields.

To quantify these observations we have measured the spatial correlation functions of the microscopic densities, i.e.

$$
n^{2} G_{a b}(\mathbf{r}, t)=\frac{1}{V} \int d \mathbf{R}\langle\delta \hat{a}(\mathbf{R}+\mathbf{r}, t) \delta \hat{b}(\mathbf{R}, t)\rangle .
$$

Here the labels $a, b$ take the values $\{n, e, \alpha, \|, \perp\}$, and refer respectively to the microscopic mass density, momentum density and local energy per particle,

$$
\begin{aligned}
\delta \hat{n}(\mathbf{r}, t) & =\sum_{i} \delta\left(\mathbf{r}_{i}(t)-\mathbf{r}\right)-n \\
n \hat{u}_{\alpha}(\mathbf{r}, t) & =\sum_{i} v_{i \alpha}(t) \delta\left(\mathbf{r}_{i}(t)-\mathbf{r}\right) \\
n \delta \hat{e}(\mathbf{r}, t) & =\sum_{i} \frac{1}{2}\left(v_{i}^{2}(t)-v_{0}^{2}(t)\right) \delta\left(\mathbf{r}_{i}(t)-\mathbf{r}\right) .
\end{aligned}
$$

The carets denote microscopic quantities. Moreover, $v_{0}(t)=\sqrt{2 T(t)}$ is the thermal velocity and $\mathbf{v}_{i}, \mathbf{r}_{i}$ are the velocity and position of the $i$-th particle. Greek labels $\alpha, \beta=\{x, y\}$ refer to Cartesian components. The second rank tensor field $G_{\alpha \beta}(\mathbf{r}, t)$ has transverse $(\perp)$ and longitudinal $(\|)$ components

$$
\begin{aligned}
G_{\|}(r, t) & =\hat{\mathbf{r}}_{\alpha} \hat{\mathbf{r}}_{\beta} G_{\alpha \beta}(\mathbf{r}, t) \\
G_{\perp}(r, t) & =\hat{\mathbf{r}}_{\perp \alpha} \hat{\mathbf{r}}_{\perp \beta} G_{\alpha \beta}(\mathbf{r}, t),
\end{aligned}
$$

where $\hat{\mathbf{r}}, \hat{\mathbf{r}}_{\perp}$ are unit vectors, parallel and perpendicular to the relative position $\mathbf{r}$. As long as the system remains spatially homogeneous with a vanishing flow velocity, 
the local energy and temperature fluctuations are the same. However, as soon as the average flow velocity $\mathbf{u}(\mathbf{r}, t)$ is nonvanishing, the granular temperature is the average energy per particle in the local rest frame.

The measurement of correlation functions in a single realization is shown in Fig. 2. The results are remarkable! Even at the earliest time $(\tau=82)$, long before any vortices are visible, long range spatial correlations have developed between the local flow velocities at different points in the fluid with a typical correlation length $\xi \simeq 30 \sigma$ ( $\xi$ is the location of the minimum in $G_{\perp}(r, t)$ ), which gradually increases to about $60 \sigma$ at $\tau=332$. The negative minimum in $G_{\perp}(r, t)$ clearly signals the presence of vortices with a diameter $\xi$. It is also interesting to compare the noise level and magnitude (vertical axes in Fig. 2) of all fluctuations, relative to the homogeneous cooling state, and correlate the signals above noise level and their growth rates with the visual observations of the $n(\mathbf{r}, t)$ - and $\mathbf{g}(\mathbf{r}, t)$-snapshots.

The dynamics of the phenomena observed seems to be controlled for a long time by the transverse flow field or vorticity modes. We conjecture that there exists an extended time regime in which these modes describe the behavior of the spatial correlation functions, at least of those correlation functions that involve the flow fields. We shall test this conjecture by analyzing the simulation results.

Moreover, we note that in normal fluids with elastic collisions, times $\tau \gtrsim 5$ are already considered long in kinetic theory. The long time tail in the velocity autocorrelation function of elastic hard spheres is typically observed in the ranges $10 \lesssim \tau \lesssim 60$. The range of the spatial correlation functions $G_{\perp}(r, t)$ and $G_{\|}(r, t)$, observed here, extends far beyond any static correlation length (a few $\sigma$ 's) and far beyond the mean free path $\left(l_{0} \simeq 0.8 \sigma\right.$ at this density).

\section{Hydrodynamic Dispersion Relations}

The previous observations on large spatial and temporal scales suggest that the long wavelength components of the microscopic fluctuations are described by linearized (because we are dealing with fluctuations) hydrodynamics. This should hold as long as fluctuations have not grown to macroscopic size. Beyond this time the evolution is controlled by the full nonlinear hydrodynamic equations.

The fluctuations discussed in the previous sections are taken relative to the homogeneous cooling state. Consequently, we linearize the hydrodynamic equations around this state, 11 and consider the Fourier components of the hydrodynamic fields $\delta n(\mathbf{k}, t), u_{\alpha}(\mathbf{k}, t) / v_{0}(t)$ and $\delta e(\mathbf{k}, t) / v_{0}^{2}(t)$ where $T(t)=\frac{1}{2} v_{0}^{2}(t)$ is the temperature in the homogeneous cooling state. The resulting set of linear equations contains still time dependent coefficients, such as the local pressure, proportional to $T(t)$, and transport coefficients, which are assumed to be independent of the dissipation parameter $\epsilon$, and proportional to the average collision frequency $\omega_{0}(t) \propto \sqrt{T(t)}$. Hence, the kinematic viscosity of inelastic hard disks is $\nu(t)=\nu \exp \left(-\gamma_{0} \tau\right)$, where $\nu=\eta / m n$ is the corresponding viscosity of elastic hard disks at the initial time.

By changing to the time variable $\tau$, introduced in Eq. (2), the linearized hydrodynamic equations transform into a set of coupled linear equations with constant 

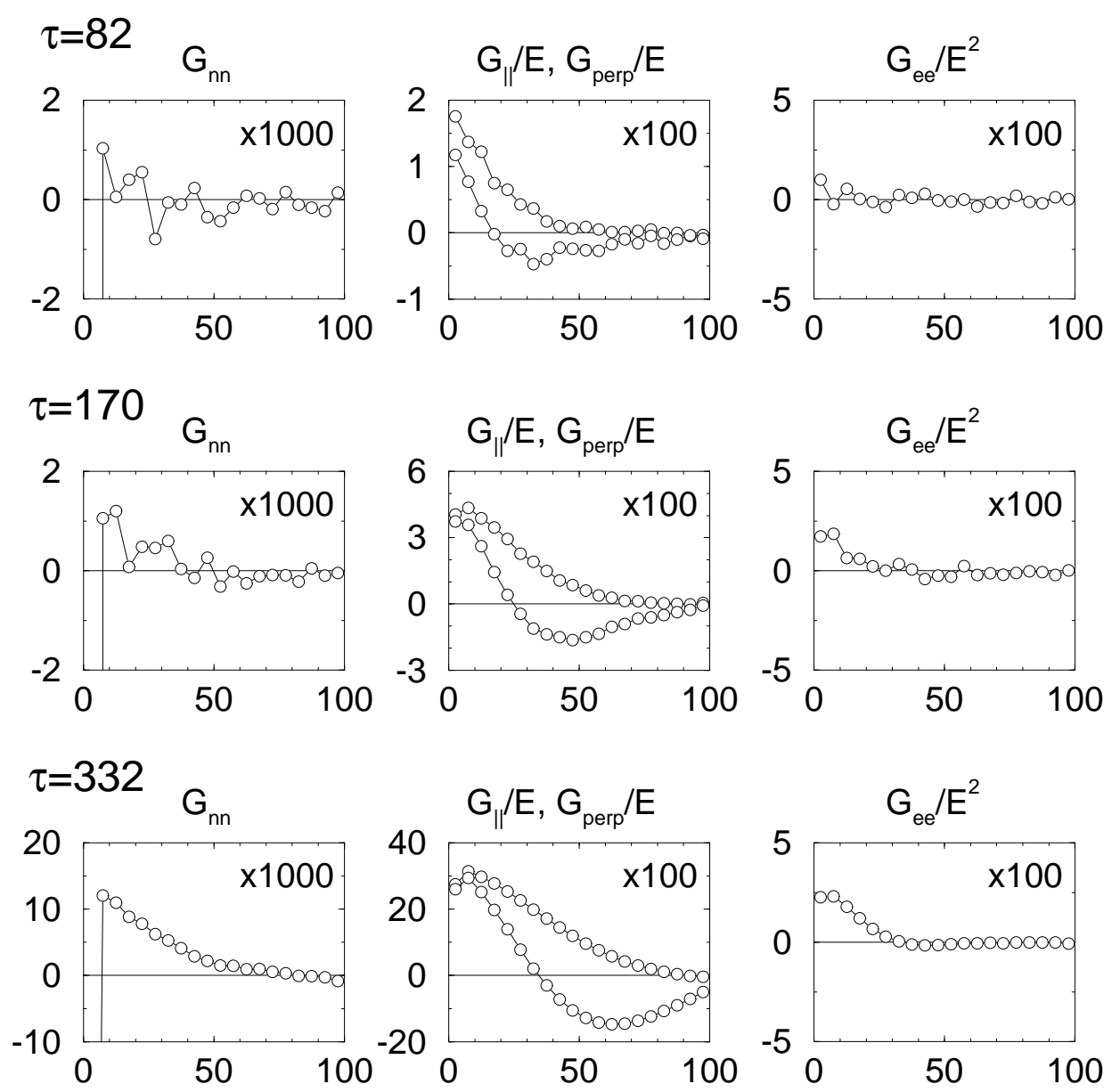

Fig. 2. Spatial correlations of relative fluctuations around the homogeneous cooling state $G_{a b}(\mathbf{r}, t) / E^{p}$ in mass densities $(n n)$, flow velocities $(\|, \perp)$ and energy densities $(e e)(p=0,1,2$ respectively), plotted versus $r / \sigma$ at a fixed number of collisions $\tau=2 C / N$ per particle, measured in a single run on a system with $N=20000$ particles in a volume $V=L^{2}$ with $L=253 \sigma$ and dissipation parameter $\epsilon=1 / 40$. Notice the vertical scales, showing magnitudes and growth rate of the fluctuations: at $\tau=332, G_{\|}$and $G_{\perp}$ have increased $(20 \times), G_{n n}(10 \times)$ and $G_{e e}(1 \times)$. The measurements of $G_{a b}(\mathbf{r}, t)$, shown here, are coarse grained by collecting data points in bins of width $\Delta r=5 \sigma$. For bin sizes of $\Delta r=0.2 \sigma$ there is already a 'signal over noise level' in $G_{\|}$and $G_{\perp}$ at $\tau=82$, whereas the signals in $G_{n n}$ and $G_{e e}$ just start to rise above noise level at short distances $r<30 \sigma$ at $\tau \simeq 220$. 
coefficients. The resulting eigenmodes have the form $\psi_{\mu}(\mathbf{k}, \tau)=\psi_{\mu}(\mathbf{k}) \exp \left[z_{\mu}(k) \tau\right]$, where $\mu=\{\perp, H, \sigma= \pm\}$ labels the shear mode $(\perp)$, the heat mode $(H)$ and the sound modes $(\sigma= \pm)$. Note that the modes only show exponential behavior if time is measured by counting collisions. In real time the modes decay algebraically. The shear mode $\psi_{\perp}(\mathbf{k}, \tau)$ is $u_{\perp}(\mathbf{k}, t) / v_{0}(t)$, and the heat and sound modes are linear combinations of the relative fluctuations $\delta n(\mathbf{k}, t), u_{\|}(\mathbf{k}, t) / v_{0}(t)$ and $\delta e(\mathbf{k}, t) / v_{0}^{2}(t)$. Here the subscripts longitudinal $(\|)$ and transverse $(\perp)$ refer to the direction of $\mathbf{k}$.

The dispersion relations for the eigenvalues $z_{\mu}$ of heat and shear mode are shown in Fig. 3 as a function of the rescaled wavenumber $q=k \sigma / \sqrt{\gamma_{0}}$. These functions

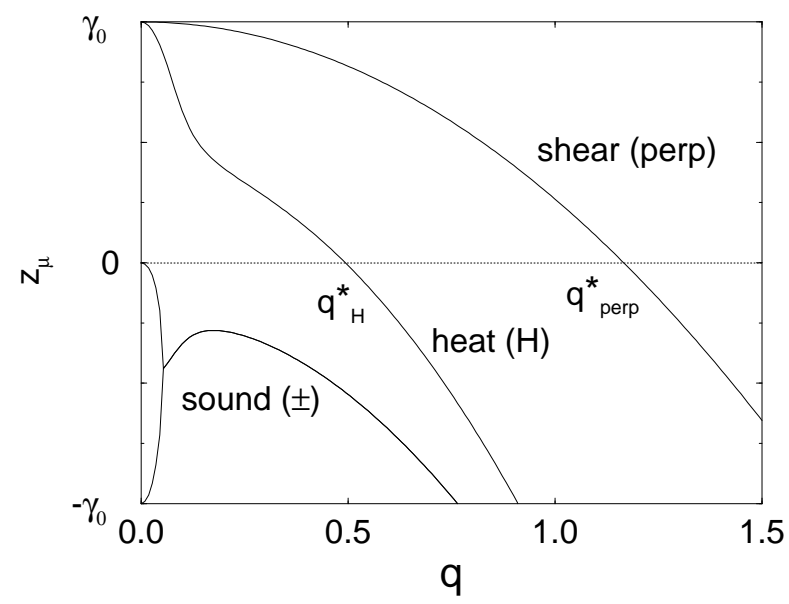

Fig. 3. Dispersion relations for the decay rates $z_{\mu}$ of hydrodynamic modes $(\mu=\{\perp, H, \pm\})$ in units $\gamma_{0}$, plotted versus the reduced wavenumber $q=k \sigma / \sqrt{\gamma_{0}}$ to make $z_{\perp} / \gamma_{0}$ independent of dissipation parameter $\gamma_{0}=\frac{1}{2} \epsilon\left(1-\frac{1}{2} \epsilon\right)$. Numerical values are calculated from the Enskog-Boltzmann equation for elastic hard disks at volume fraction $\phi=\frac{1}{4} \pi n \sigma^{2} \simeq 0.245$. Wavenumber $q_{H}^{*}$ and $q_{\perp}^{*}$ are the thresholds of stability for heat and shear modes.

intersect the $q$-axis respectively at $q_{H}^{*}$ and $q_{\perp}^{*}$, where $q_{\mu}^{*}=k_{\mu}^{*} \sigma / \sqrt{\gamma_{0}}$. At volume fraction $\phi \simeq 0.245$ one has $q_{H}^{*} \simeq 0.50$ and $q_{\perp}^{*} \simeq 1.17$. The numerical values of the transport coefficients are calculated from the Enskog theory for elastic hard disks 12 at a volume fraction $\phi \simeq 0.245$ and initial temperature $T(0)=1$, used in the present simplations. We only quote the result for the shear mode or transverse velocity field, 19.11 which decays as

$$
\begin{aligned}
u_{\perp}(\mathbf{k}, t) & \sim v_{0}(t) \exp \left[z_{\perp}(k) \tau\right] \\
& =v_{0}(0)\left(1+t / t_{e}\right)^{-\nu k^{2} t_{e}}
\end{aligned}
$$

where the dispersion relation for the relaxation rate is:

$$
z_{\perp}(k)=\gamma_{0}\left(1-\nu k^{2} t_{e}\right)=\gamma_{0}\left(1-\hat{\nu} q^{2}\right),
$$

with $\hat{\nu}=\nu t_{0} / \sigma^{2}$. If the viscosity of the inelastic hard disks depend on the dissipation 
parameter $\epsilon$ only through $\sqrt{T(t)}$ - as we have assumed here - then $z_{\perp}(k) / \gamma_{0}$ is a universal function of $q=k \sigma / \sqrt{\gamma_{0}}$, independent of the dissipation parameter $\gamma_{0}$.

\section{Instabilities and Clustering}

The spectrum of eigenvalues in Fig. 3 shows that the relative fluctuations with respect to the homogeneous cooling state contain several unstable modes with an exponential growth rate, $\exp \left[z_{\mu}(k) \tau\right]$, if time is measured in collision numbers $\tau$. These unstable modes, $\psi_{\mu}(\mathbf{k}, \tau)$, are the long wavelength shear mode $(\mu=\perp)$ with $k<k_{\perp}^{*}$, and the long wavelength heat mode $\psi_{H}(k, \tau)$ with $k<k_{H}^{*}<k_{\perp}^{*}$. The sound modes are stable for all wavelengths. The dynamics of these slow modes determines the time evolution of the long wavelength fluctuations, away from the homogeneous cooling state, and determines a mechanism for initial pattern selection. In the initial stages spontaneous fluctuations with respect to the homogeneous cooling state occur on all possible wavelengths. Those with $k$ smaller than $k_{H}^{*}$ or $k_{\perp}^{*}$ will grow at an exponential rate $\exp \left[z_{\mu}(k) \tau\right]$.

The fastest growing mode is the one with the smallest wavenumber, as can be seen from the dispersion relations in Fig. 3. In finite systems with periodic boundary conditions, as used in the present computer simulations, the smallest wavenumber allowed is $k_{m}=2 \pi / L$. As long as $k_{m}<k_{H}^{*}$, unstable shear modes as well as heat modes can be excited, and we can estimate the onset times $\tau_{\mu}$ for the shear and heat mode instabilities by the criterion

$$
z_{\mu}\left(k_{m}\right) \tau_{\mu} \simeq 1 \quad(\mu=\perp, H)
$$

which implies that the amplitude of relative fluctuation has increased by a factor $e$ above its normal level. As long as the fluctuations are small and, hence, can be described by linearized hydrodynamic equations, the vorticity mode does not couple to density and energy fluctuations, and the onset for the appearance of vortex structure is the time $\tau_{\perp}$, as defined in Eq. (8). The fluctuations in mass and energy densities, however, do couple to the unstable heat mode, and the onset time for observable inhomogeneities in the mass density (clustering) and energy field will be of order $\tau_{H}$.

For the system used in the simulations of section 3 with $L=253 \sigma$ and $\epsilon=0.025$ one has $q_{m} \simeq 0.22$. The corresponding rate constants $z_{\mu}\left(k_{m}\right)$ can be read off from Fig. 3 and yield estimates of the onset times for the appearance of vorticity $\left(\tau_{\perp} \simeq 83\right)$ and for clustering in the density $\left(\tau_{H} \simeq 225\right)$. Inspection of the configurations $n(\mathbf{r}, t)$ and $\mathbf{g}(\mathbf{r}, t)$, as well as the correlations in Fig. 2, shows that the structures in $G_{n n}(r, t)$ and $G_{e e}(r, t)$ are barely noticeable at $\tau=170$, and well developed at $\tau=332$, consistent with the theoretical estimates for $\tau_{\perp}$ and $\tau_{H}$.

Suppose we decrease the system size or decrease $\epsilon$, such that $q_{m}=2 \pi \sigma /\left[L \sqrt{\gamma_{0}}\right]$ increases and moves into the interval $\left(q_{H}^{*}, q_{\perp}^{*}\right)$. Then only shear modes can be excited and the onset time $\tau_{\perp}$ for the appearance of vortex structures will increase like $1 / z_{\perp}\left(k_{m}\right)$ with increasing $q_{m}$. The vorticity will grow, but density and energy fields will remain spatially homogeneous, as long as linearized hydrodynamics is 
adequate. An example of such a system is realized at $N=5000, L=126 \sigma$ and $\epsilon=0.007$, where $q_{m} \simeq 0.84$, which is located in the middle of the interval $\left(q_{H}^{*}, q_{\perp}^{*}\right)$. The simulations show that the total energy follows the homogeneous cooling law, $E(t)=E(0) \exp \left[-2 \gamma_{0} \tau\right]$, up to $\tau \simeq 1100$. Of course, in all cases where $k_{m}<k_{\perp}^{*}$, eventually the fluctuations will grow to macroscopic size, and after a very long time nonlinear hydrodynamics takes over, 2 and leads again to inhomogeneities in densityand energy fields.

If one still further increases $q_{m}$, it will pass the stability threshold $q_{\perp}^{*}$, and none of the unstable modes is excitable. All hydrodynamic modes become stable, and the system will remain in the homogeneous cooling state for all times. McNamara and Young $E$ call this state the kinetic state. An example is the system $\{N=5000, L=$ $126 \sigma, \epsilon=0.001\}$, where $q_{m} \simeq 2.2>q_{\perp}^{*}$. Even after $\tau=2044$ no inhomogeneities in density or flow fields have developed, and the homogeneous cooling law is still obeyed, as can be inferred from the measured correlation functions $G_{a b}(\mathbf{r}, t)$ and the measured kinetic energy $E(t)$.

\section{Vorticity Diffusion and Scaling}

A rough estimate of the behavior of the pair correlation function can be obtained from the Cahn-Hilliard theory 13 for the dynamics of phase separation. In this theory one calculates the structure factors, defined as the Fourier transforms $S_{a b}(\mathbf{k}, t)=$ $V^{-1}\langle\delta \hat{a}(\mathbf{k}, t) \delta \hat{b}(\mathbf{k}, t)\rangle$ of the correlation functions $G_{a b}(\mathbf{r}, t)$, from the long wavelength behavior of the unstable hydrodynamic (macroscopic) modes $\delta a(\mathbf{k}, t)$. Very recently Deltour and Barrat used this theory to calculate the structure factor $S_{n n}$ for a fluid of inelastic hard disks. 14

Here a similar estimate for the structure factor $S_{\perp}(k, t)=V^{-1}\left\langle\left|\hat{u}_{\perp}(\mathbf{k}, t)\right|^{2}\right\rangle$ of the vorticity field yields $S_{\perp}(k, t) \propto v_{0}^{2}(t) \exp \left[2 z_{\perp}(k) \tau\right] \propto \exp \left[-2 \nu t_{0} k^{2} \tau\right]$. On the basis of the conjecture, formulated in section 3 , the contributions of heat and sound modes can be neglected. The correlation functions $G_{\perp}(r, t)$ and $G_{\|}(r, t)$ can then be obtained by taking inverse Fourier transforms of $(\hat{\mathbf{k}} \cdot \hat{\mathbf{r}})^{2} S_{\perp}(k, t)$ and $\left[1-(\hat{\mathbf{k}} \cdot \hat{\mathbf{r}})^{2}\right] S_{\perp}(k, t)$ respectively, where $\hat{\mathbf{a}}=\mathbf{a} /|\mathbf{a}|$. They have the generic scaling form $\tau^{-1} F_{\mu}\left(r / \sqrt{\nu t_{0} \tau}\right)$ with $\mu=\{\perp, \|\}$. The explicit form of $F_{\mu}(x)$ is not used in the arguments presented below.

We recall that the average diameter $\xi(t)$ of a vortex can be identified with the location of the minimum of $G_{\perp}(r, t)$. It then follows from the scaling form and Eq. (2) that

$$
\xi(t) \simeq a_{0} \sqrt{\nu t_{0} \tau} \propto \sqrt{\nu t_{e} \ln \left(t / t_{e}\right)},
$$

where the constant of proportionality $a_{0}$ is independent of the volume fraction $\phi$ and of the dissipation parameter $\epsilon$. For large systems the value of $a_{0}$, measured in the simulations, is typically $a_{0} \simeq 4.0-5.3$ or $3.8-4.0$ in the $\tau$-intervals $(10-80)$ or $(82-216)$ respectively for systems with parameters $\left\{N=5 \times 10^{4}, \epsilon=0.1, \phi=0.4\right\}$ or $\left\{N=2 \times 10^{4}, \epsilon=0.025, \phi=0.245\right\}$. For small systems (typically $N=5000$, $\epsilon<0.1), \xi(t)$ saturates to a constant value and diffusive growth is absent due to 
interference effects caused by the periodic boundaries.

The diffusive growth of the average vortex diameter offers strong support for the validity of our conjecture that the long time dynamics on large spatial scales is mainly governed by vorticity diffusion. More refined Ring kinetic theory or mode coupling theories provide detailed information 15 about the explicit analytic forms of $G_{\mu}(r, t)$. It is found that data collapse for $G_{\mu}(r, t)$ with $\mu=\{\perp, \|\}$ only hold for large distances, where $r>\sqrt{\nu t_{0} \tau}$, and large times, where $\tau>\tau_{e}=(\ln 2) / \gamma_{0}$. Here $\tau_{e} \equiv \tau\left(t_{e}\right)$ is the characteristic time scale for homogeneous cooling, as defined through Eq. (2).

We conclude that our theoretical analysis has provided a consistent picture of the long range dynamic correlations that have built up through the dissipative dynamics of inelastic hard sphere systems. This dynamics violates the conditions of detailed balance.

The theoretical analysis also predicts the existence of algebraic long range correlations $\propto 1 / r^{d}$, where $d$ is the dimensionality of the hard sphere system. Similar algebraic tails have been observed in lattice gas automata with collision rules that violate the conditions of detailed balance. 6 The complete analysis and theoretical predictions, which have no adjustable parameters, as well as extensive comparisons with the results of computer simulations will be reported elsewhere.15

A comparison with the simulation results for a single realization is shown in Fig. 4.

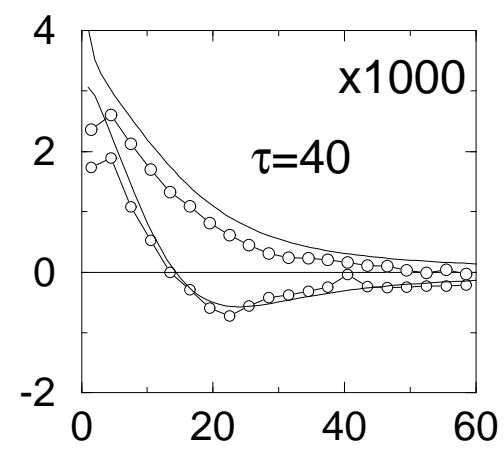

Fig. 4. Comparison of theory (solid line) and simulations (circles) of $G_{\|}(r, t)$ and $G_{\perp}(r, t)$ as functions of $r / \sigma$ at $\tau=2 C / N \simeq 40$ collisions per particle in a system with $N=5000, L=126 \sigma$, dissipation parameter $\epsilon=0.1$, homogeneous cooling length $l_{e} \simeq 7 \sigma$, with unstable heat and shear modes $\left(q_{m}=2 \pi \sigma /\left[L \sqrt{\gamma_{0}}\right] \simeq 0.23<q_{H}^{*}\right)$, and estimated transition to the shearing state at $\tau_{\operatorname{tr}}=L^{2} /\left[64 \nu t_{0}\right] \simeq 325$.

There is a quantitative agreement between theory and simulations for all $r$ values, provided $\tau$ is sufficiently small $(\tau \lesssim 60$ in Fig. 4$)$ to avoid effects from nonlinear hydrodynamics, and provided system size $L$ and/or dissipation parameter $\epsilon$ are sufficiently large to avoid interference effects from periodic boundaries. 


\section{Transition to Shearing States/Boundary Effects}

What are the asymptotic states observed in computer simulations? In large but finite systems with $L$ and $\epsilon$ large enough (that is $q_{m}<q_{H}^{*}$ ), so that all unstable modes can be excited, the mean vortex diameter can not grow indefinitely. It will typically stop growing at a transition time $\tau_{\text {tr }}$, where $\xi\left(\tau_{\text {tr }}\right) \simeq \frac{1}{2} L$, and the periodic boundaries force a transition to an inhomogeneous shearing state. The dynamics of this transition, caused by fusion of vortices of equal sign into shearing layers, is well illustrated in the snapshot of the flow field in Fig. 10b of Ref. 10. In this case, the transition time $\tau_{\text {tr }}$ can be estimated from the growth law $\xi(\tau) \simeq 4 \sqrt{\nu t_{0} \tau}$, and yields $\tau_{\mathrm{tr}} \simeq L^{2} / 64 \nu t_{0}$, which is proportional to the volume of the system, and independent of the dissipation parameter $\epsilon$. For the system with $\{N=5000, L=126, \epsilon=0.1\}$ the theoretical estimate gives $\tau_{\mathrm{tr}} \simeq 325$, whereas the simulations yield $\tau_{\mathrm{tr}} \simeq 400 \pm 25$. In the system of Ref. 2 with $\left\{N=4 \times 10^{4}, L \simeq 793, \epsilon=0.4\right\}$ the above estimate gives $\tau_{\text {tr }} \simeq 525$, whereas the value reported for the simulation is $\tau_{\text {tr }}=2 C / N \simeq 600$.

However, for systems with $\{N=5000, L \simeq 126, \epsilon \lesssim 0.01\}$, and for the small systems of Ref. 10 with $\{N=1024, L=57, \epsilon \simeq 0.18-0.45\}$ diffusive growth of the mean vortex diameter is absent due to interference effects resulting from the periodic boundaries, as the stability wavelength for the heat mode $l_{H}=2 \pi / k_{H}^{*}$ is of order $L$ (see discussion below). As diffusive growth is absent, the transition time to the shearing state, $\tau_{t r}=2 C / N \simeq 1400-1600$, observed in the simulations of Fig. 5, and of Ref. 10, has no connection to the theoretical estimate $\tau_{t r}=L^{2} / 64 \nu t_{0}$, valid for large systems.

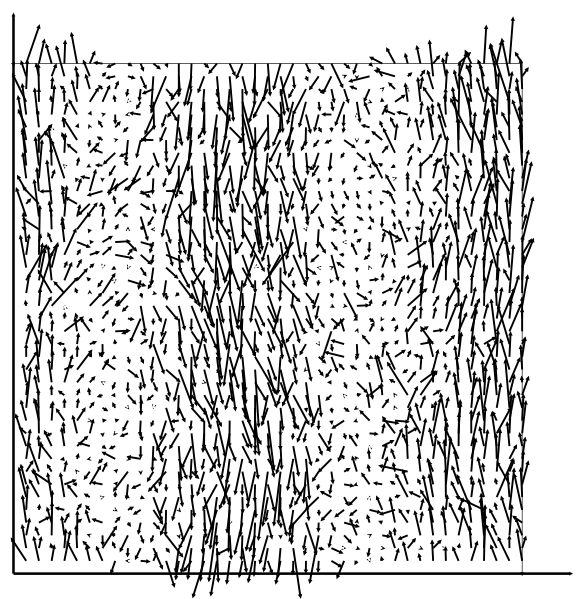

Fig. 5. Flow field, coarse grained over cells of $3 \sigma \times 3 \sigma$, showing a transition at $\tau_{\operatorname{tr}}=2 C / N \simeq 1600$ collisions per particle to a shearing state with a homogeneous density distribution in a small system with $N=5000, L=126 \sigma$, dissipation parameter $\epsilon=0.01$, stability wavelength $l_{H} \simeq 126 \sigma$, and homogeneous cooling length $l_{e} \simeq 160 \sigma$ (see section 7 ).

In the inelastic hard sphere system under discussion, there exists a number of intrinsic dynamical length scales: the mean free path $l_{0}$, the homogeneous cooling 
length $l_{e}=v_{0}(0) t_{e}=l_{0} / \gamma_{0}$; the mean vortex size $\xi(t)$; the stability wavelength, $l_{\mu}=2 \pi / k_{\mu}^{*}$, for the stability of the heat $(\mu=H)$ and shear mode $(\mu=\perp)$.

If any of these length scales become of the order of the system size $L$, the effects of the boundaries dominate the behavior of the system. If one is dealing with finite geometries, the effects of physical boundaries become important, and one needs to model particle-wall interactions.

In case one is dealing with computer simulations, in which periodic boundary conditions are used, the intrinsic length scales should be less than $\frac{1}{3} L$, say, in order to minimize finite size effects. If, however, one of these length scales becomes of order $L$, the long time dynamics of the system is totally controlled by the artificial periodic boundary conditions, and the final states are artifacts of these unphysical boundaries.

The above discussions show that the homogeneous and inhomogeneous shearing states, as well as the kinetic state, discussed in the previous sections, and reported in the literature 210.4 do not represent physical properties of macroscopic (idealized) granular flows, but are caused by the artificial periodic boundary conditions. It is also interesting to observe that in cases where the boundary conditions have been modelled in a physically more realistic way and in different geometries 16 i.e. by a wall kept at a constant temperature - different asymptotic states have been observed. This discussion suggests that more realistic modelling of collisions between granular particles and physical walls is imperative for understanding the asymptotic states of granular flows contained in finite geometries.

\section{Acknowledgements}

Two of us (R.B. \& T.v.N.) acknowledge support of the foundation 'Fundamenteel Onderzoek der Materie (FOM)', which is financially supported by the Dutch National Science Foundation (NWO). One of us (R.B.) acknowledges further financial support from Universidad Complutense through the program 'Estancias Breves en el Extranjero' and DGICYT (Spain) number PB94-0265. One of us (M.H.E.) acknowledges support from the Offices of International Relations of Universidad Complutense and Universiteit Utrecht for his stay in Madrid.

\section{References}

1. J.T. Jenkins and M.W. Richman, Phys. Fluids 28, 3485 (1985).

2. I. Goldhirsch and G. Zanetti, Phys. Rev. Lett. 70, 1619 (1993), I. Goldhirsch, M-L. Tan and G. Zanetti, J. Scient. Comp. 8, 1 (1993).

3. M.P. Allen and D.J. Tildesley, Computer Simulation of Liquids (Clarendon Press, Oxford, 1987).

4. J.R. Dorfman and T.R. Kirkpatrick, in Systems Far from Equilibrium, ed. L. Garrido (Lecture Note in Physics 132, Springer Verlag, Berlin, 1980), p. 263.

5. J.R. Dorfman, T.R. Kirkpatrick and J. Sengers, Ann. Rev. Phys. Chem. 45, 213 (1994).

6. H.J. Bussemaker and M.H. Ernst, Phys. Rev. E 53, 5837 (1996). 
7. Conference/Workshops in Zaragoza - May 1996, Lyon - July 1996, Boston - August 1996 and Cambridge - September 1996 (unpublished).

8. A. Goldshtein and M. Shapiro, J. Fluid Mech. 282, 75 (1995).

9. J.J. Brey, F. Moreno and J.W. Dufty, preprint (1996).

10. S. McNamara and W.R. Young, Phys. Rev. E 53, 5089 (1996).

11. S. McNamara, Phys. Fluids A 5, 3056 (1993).

12. S. Chapman and T.G. Cowling, The Mathematical Theory of Non-uniform Gases, (Cambridge University Press, 1970).

13. J.S. Langer, in Solids Far from Equilibrium, ed. C. Godrèche (Cambridge University Press, 1992), p. 297.

14. P. Deltour and J.-L. Barrat, preprint cond-mat/9609184.

15. T.P.C. van Noije, M.H. Ernst, J.A.G. Orza and R. Brito, to be published.

16. S.E. Esipov and T. Pöschel, preprint cond-mat/9609096. 